J. Dairy Sci. 104:7343-7343

https://doi.org/103168/jds.2021-104-6-7343

() 2021 American Dairy Science Association ${ }^{\circledR}$. Published by Elsevier Inc. and Fass Inc. All rights reserved.

\title{
Corrigendum to "Addition of straw to the early-lactation diet: Effects on feed intake, milk yield, and subclinical ketosis in Holstein cows" (J. Dairy Sci. 104:3008-3017)
}

\section{Hesam A. Seifi, Julianna M. Huzzey, M. A. Khan, Daniel M. Weary, and Marina A. G. von Keyserlingk}

In Table 1 (page 3010), the authors presented the calculated ration formulation and not the analytical values of the ration composition. The revised table is shown below with corrected values in bold.

The authors regret the error.

\section{REFERENCES}

Seifi, H. A., J. M. Huzzey, M. A. Khan, D. M. Weary, and M. A. G. von Keyserlingk. 2021. Addition of straw to the early-lactation diet: Effects on feed intake, milk yield, and subclinical ketosis in Holstein cows. J. Dairy Sci. 104:3008-3017. https://doi.org/10.3168/jds.2020-18549.

Table 1. Composition and component analysis (\% of DM unless otherwise noted) of composite samples of 3 TMR types fed to transition cows: dry-cow TMR (DTMR), conventional postpartum TMR (CTMR), and postpartum TMR with added wheat straw (STMR; fed only during the 3 -wk postcalving treatment period $)^{1}$

\begin{tabular}{lrrr}
\hline Composition & DTMR & CTMR & STMR \\
\hline Ingredient & & & \\
Grass silage & 13.07 & 22.25 & 21.00 \\
Corn silage & 30.25 & 16.64 & 15.93 \\
Alfalfa hay & 16.43 & 11.72 & 11.22 \\
Concentrate mix ${ }^{2}$ & 14.17 & 49.38 & 47.27 \\
Wheat straw & 26.08 & 0.00 & 4.27 \\
Component & & & \\
DM & 49.28 & 52.45 & 53.41 \\
NE (Mcal/kg) & $\mathbf{1 . 4 5}$ & $\mathbf{1 . 5 7}$ & $\mathbf{1 . 5 7}$ \\
TDN & $\mathbf{6 3 . 7 5}$ & $\mathbf{6 8 . 6 3}$ & $\mathbf{6 8 . 6 7}$ \\
CP & $\mathbf{1 3 . 5 6}$ & $\mathbf{1 6 . 0 9}$ & $\mathbf{1 6 . 5 8}$ \\
NDF & $\mathbf{4 4 . 3 4}$ & $\mathbf{3 7 . 5 5}$ & $\mathbf{3 8 . 1 1}$ \\
ADF & $\mathbf{3 2 . 2 9}$ & $\mathbf{2 6 . 0 2}$ & $\mathbf{2 5 . 9 7}$ \\
NFC & $\mathbf{3 0 . 6 0}$ & $\mathbf{3 4 . 8 6}$ & $\mathbf{3 3 . 8 0}$ \\
Starch & $\mathbf{1 1 . 5 7}$ & $\mathbf{1 5 . 5 3}$ & $\mathbf{1 6 . 8 5}$ \\
Crude fat & $\mathbf{2 . 1 8}$ & $\mathbf{2 . 9 3}$ & $\mathbf{2 . 8 9}$ \\
Ash & $\mathbf{8 . 3 4}$ & $\mathbf{9 . 5 0}$ & $\mathbf{9 . 2 4}$ \\
Ca & $\mathbf{0 . 9 4}$ & $\mathbf{1 . 0 5}$ & $\mathbf{0 . 9 1}$ \\
P & $\mathbf{0 . 2 6}$ & $\mathbf{0 . 3 3}$ & $\mathbf{0 . 3 4}$ \\
Mg & $\mathbf{0 . 3 2}$ & $\mathbf{0 . 3 1}$ & $\mathbf{0 . 3 0}$ \\
Na & $\mathbf{0 . 1 6}$ & $\mathbf{0 . 3 8}$ & $\mathbf{0 . 3 7}$ \\
K & $\mathbf{1 . 5 7}$ & $\mathbf{1 . 7 8}$ & $\mathbf{1 . 8 0}$ \\
S & $\mathbf{0 . 1 9}$ & $\mathbf{0 . 2 2}$ & $\mathbf{0 . 2 1}$ \\
\hline
\end{tabular}

${ }^{1}$ All cows were fed the CTMR after the treatment period $(\mathrm{n}=34$ cows per treatment).

${ }^{2}$ For DTMR, the major ingredients included $45 \%$ Amino Plus (Ag Processing Inc., Omaha, NE), $12 \%$ corn gluten meal, $11 \%$ soybean meal, $7.36 \%$ fine limestone, $5 \%$ ground beet pulp, $5 \%$ dry cow mineral, $4.32 \%$ calcium sulfate, $3.544 \%$ distillers corn wheat blend, $1 \%$ molasses. CTMR and STMR: 34\% rolled barley, 20\% fine ground barley, $10 \%$ distillers corn wheat blend, $7.3 \%$ ground wheat, $6 \%$ soybean meal, $6 \%$ Amino Plus (Ag Processing Inc.), $5 \%$ canola meal, $2.22 \%$ fine limestone, $1.18 \%$ mill run pellets. (Analysis conducted by A\&L Canada Laboratories Inc., London, ON, Canada.) 